\title{
In Potential Stroke Patients on Warfarin, the International Normalized Ratio Predicts Ischemia
}

\author{
Cathy Cao ${ }^{a} \quad$ Ashley Martinelli ${ }^{b}$ Brian Spoelhof ${ }^{b}$ Rafael H. Llinas ${ }^{c}$ \\ Elisabeth B. Marsh ${ }^{\mathrm{c}}$ \\ a Department of Pharmacy, The Johns Hopkins Hospital, Baltimore, MD, USA; \\ ${ }^{b}$ Department of Pharmacy, Johns Hopkins Bayview Medical Center, Baltimore, MD, USA; \\ 'Department of Neurology, Johns Hopkins School of Medicine, Baltimore, MD, USA
}

\author{
Keywords \\ Warfarin · Ischemic stroke $\cdot$ International normalized ratio $\cdot$ Cerebrovascular disease
}

\begin{abstract}
Background: Stroke can occur in patients on warfarin despite anticoagulation. Patients with a low international normalized ratio (INR) should theoretically be at greater risk for ischemia than those who are therapeutic. Therefore, INR may be able to indicate whether new neurological deficits are more likely strokes or stroke mimics in patients on warfarin. This study evaluates the association and predictive value of INR in determining the likelihood of ischemia. Methods: Patients were identified using the acute stroke registry at a Primary Stroke Center from January 2013 through December 2014. All adult patients undergoing evaluation for acute stroke with prior documented use of warfarin and an INR level at presentation were included. Data were collected regarding patient demographics, medical comorbidities, stroke severity, reason for anticoagulation, and laboratory studies including INR. Student $t$ tests and $\chi^{2}$ analysis were used to evaluate factors associated with increased likelihood of ischemia (stroke or transient ischemic attack) versus mimic. Significant results were entered into a multivariable regression analysis. Sensitivity and specificity analyses were conducted to determine the predictive value of INR for ischemic risk. Results: 116 patients were included; 46 were diagnosed with ischemia, 70 were diagnosed as mimics. $75 \%$ of patients were on warfarin for atrial fibrillation versus $25 \%$ for venous thrombosis. A statistically significant difference in mean INR for patients with ischemia $(n=46)$ versus mimics $(n=70)$ was observed (1.7 vs. 2.8; $p<0.001)$. In multivariable analysis, both sub-therapeutic INR $(p<0.001)$ and atrial fibrillation
\end{abstract}


( $p=0.014$ ) were predictors of ischemia. In patients with an INR $\geq 2$, the predictive value of having a non-ischemic etiology was $79 \%$. No patient with an INR of $\geq 3.6$ was found to have ischemia. Conclusions: Sub-therapeutic INR and atrial fibrillation are strongly associated with ischemia in patients on warfarin presenting with acute neurologic symptoms. Ischemia is far less likely in patients with an INR of $\geq 2$ and rare in those with an INR $\geq 3.6$. This study shows that the INR value of a patient on warfarin can help stratify patients' risk for acute ischemic stroke and guide further neurologic imaging and workup.

(C) 2017 The Author(s)

Published by S. Karger AG, Basel

\section{Introduction}

Stroke is the fifth leading cause of mortality in the United States. Nearly $90 \%$ of all stroke cases are ischemic in nature [1]. Rapid assessment of patient eligibility for intravenous recombinant tissue plasminogen activator (rt-PA) is critical in these cases to re-establish blood flow to the ischemic area [2-4]. The use of warfarin by itself is not an absolute contraindication to rt-PA administration; though due to increased bleeding risk, an international normalized ratio (INR) of $>1.7$ is a contraindication [5]. Because of this, tests of coagulation are included in the acute workup of patients on anticoagulation who present with new neurologic deficits [6]. Another reason to obtain an INR on admission in these patients may be its ability to help determine the likelihood of ischemia.

There is little published data regarding the incidence of ischemic stroke in patients already on warfarin with varying INR cutoffs, or the predictive value of INR in informing the likelihood of ischemia versus a stroke mimic. For the majority of patients on warfarin, available guidelines recommend an INR goal of 2-3 [7]. Unfortunately, patients are often subtherapeutic given the difficulty in controlling INR due to medication and food interactions [8, 9]. For many indications, such as atrial fibrillation or a hypercoagulable state, a sub-therapeutic INR could increase risk for an ischemic stroke [10]. The aim of the present study is to determine the likelihood of ischemia (stroke or transient ischemic attack [TIA]) in patients on warfarin who present with stroke-like symptoms, based on their INR result.

\section{Materials and Methods}

\section{Patient Selection}

A retrospective analysis was performed of all patients presenting to our urban, academic stroke center with symptoms suggestive of acute stroke within the last $6 \mathrm{~h}$, who were also on warfarin, between January 1, 2013, and December 31, 2014. The study was approved by the Institutional Review Board of the Johns Hopkins Health System. Eligible patients included adults (age $\geq 18$ years), with warfarin documented as home medication, who had an INR obtained at presentation, and were included in our Brain Attack Registry. This registry is maintained by our Stroke Program to track patients presenting to the Emergency Department with symptoms concerning for acute stroke. Patients were excluded if they failed to meet the above criteria, had lack of further follow-up information regarding diagnosis, or were on warfarin because of mechanical valve (different INR goal: 2.5-3.5).

Patients were evaluated by a neurologist as part of the "brain attack (BAT)" or acute stroke code. They were categorized by final diagnosis using history, examination, laboratory data, and neuroimaging (MRI and non-contrast head CT) into ischemic (ischemic stroke or TIA) versus non-ischemic (hemorrhagic stroke or stroke mimic [other determined diagnosis]). In addition to demographic information, admission INR, and indication for warfarin 
Cao et al.: In Potential Stroke Patients on Warfarin, the International Normalized Ratio Predicts Ischemia

Fig. 1. Study flow diagram.

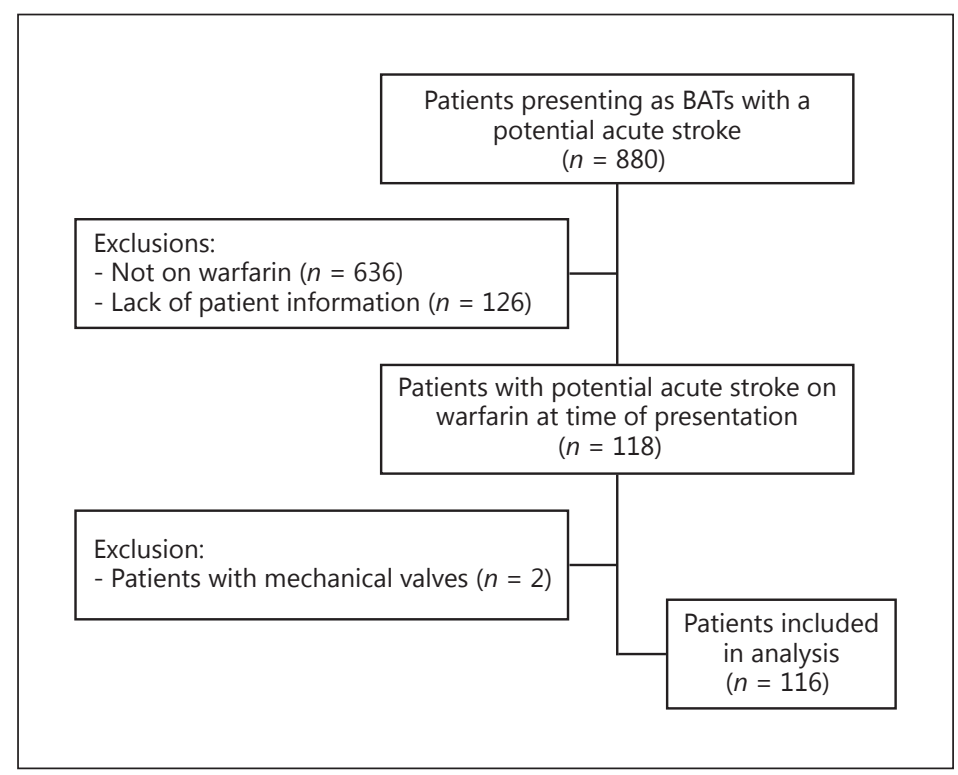

therapy; baseline laboratory results, stroke characteristics when applicable (Trial of Org 10172 in Acute Stroke Treatment [TOAST] criteria), thrombolytic administration, and outcome (discharge modified Rankin scale score [mRS]) data were collected. The primary objective was to determine the association between initial INR and ischemia in patients on warfarin presenting with acute neurologic deficits. The secondary objective was to compare outcomes in patients with ischemic stroke who presented with a therapeutic versus subtherapeutic INR. We hypothesized that those with a therapeutic INR would be less likely to have ischemia than individuals who were sub-therapeutic, and that when they did present with strokes, they would be less severe.

\section{Analysis}

Statistical analyses were performed using Stata ${ }^{\circledR}$ (version 13·2, College Park, TX, USA). Continuous and normally distributed variables were compared using Student $t$ tests. Categorical and nominal variables were calculated using $\chi^{2}$ tests. A $p$ value of $<0.05$ was considered to be statistically significant. Multivariable regression was used to determine the odds ratio for any variable significant in univariate analysis and those predetermined to be potentially clinically significant (age, race, sex). A subsequent sensitivity and specificity analysis was performed to evaluate the relationship between INR and ischemic risk. Positive and negative predictive values were determined for multiple INR cut-points. In a secondary analysis, patients presenting with hemorrhage were removed from the "non-ischemic" group and analyses were repeated.

\section{Results}

\section{Patients and Baseline Characteristics}

One-hundred eighteen patients met inclusion/exclusion criteria for this study (Fig. 1). Two additional patients were excluded due to indication for anticoagulation (mechanical valve). The baseline characteristics of the 116 patients are shown in Table 1 . The median age was 77 years; $57 \%$ were women; $74 \%$ were white. Indications for warfarin therapy could be 
Cao et al.: In Potential Stroke Patients on Warfarin, the International Normalized Ratio Predicts Ischemia

Table 1. Patient characteristics: predictors of stroke in patients on warfarin

\begin{tabular}{|c|c|c|c|c|}
\hline & $\begin{array}{l}\text { Total } \\
(n=116)\end{array}$ & $\begin{array}{l}\text { Ischemia } \\
(n=46)\end{array}$ & $\begin{array}{l}\text { Non-ischemia } \\
(n=70)\end{array}$ & $p$ value \\
\hline \multicolumn{5}{|l|}{ Demographic information } \\
\hline Median age (IQR), years & $77(67-84)$ & $77(67-82)$ & $77(67-84)$ & 0.830 \\
\hline Race, $n(\%)$ & & & & 0.716 \\
\hline White & $86(74)$ & $35(76)$ & $51(73)$ & - \\
\hline Black & $23(20)$ & $9(20)$ & $14(20)$ & - \\
\hline Asian & $2(2)$ & $0(0)$ & $2(3)$ & - \\
\hline Women, $n(\%)$ & $66(57)$ & $28(61)$ & $38(54)$ & 0.484 \\
\hline Smoking, $n(\%)$ & $26(23)$ & $9(20)$ & $17(25)$ & 0.497 \\
\hline Alcohol, $n(\%)$ & $26(23)$ & $6(13)$ & $20(29)$ & 0.041 \\
\hline \multicolumn{5}{|l|}{ Present medical history, $n(\%)$} \\
\hline Coronary artery disease & $74(64)$ & $30(65)$ & $44(64)$ & 0.874 \\
\hline History of stroke & $54(47)$ & $21(46)$ & $33(47)$ & 0.875 \\
\hline History of TIA & $36(31)$ & $14(30)$ & $22(31)$ & 0.910 \\
\hline Chronic heart failure & $38(33)$ & $15(33)$ & $23(33)$ & 0.978 \\
\hline Hypertension & $110(95)$ & $44(96)$ & $66(94)$ & 0.745 \\
\hline Diabetes mellitus & $46(40)$ & $17(37)$ & $29(41)$ & 0.630 \\
\hline \multicolumn{5}{|l|}{ Warfarin information } \\
\hline Mean initial INR (SD) & $2.4(1.5)$ & $1.7(0.7)$ & $2.8(1.7)$ & $<0.001$ \\
\hline Arterial indication for warfarin, $n(\%)^{1}$ & $88(76)$ & $40(87)$ & $48(67)$ & 0.024 \\
\hline \multicolumn{5}{|l|}{ Laboratory values } \\
\hline Mean platelets (SD), $10^{3} / \mathrm{mm}^{3}$ & $230(85)$ & $252(99)$ & $215(71)$ & 0.021 \\
\hline Mean LDL (SD), mg/dL & $75(30)$ & $78(33)$ & $70(22)$ & 0.309 \\
\hline Mean SBP (SD), mm Hg & $154(28)$ & $155(27)$ & $152(29)$ & 0.533 \\
\hline Mean DBP (SD), $\mathrm{mm} \mathrm{Hg}$ & $84(16)$ & $85(15)$ & $82(17)$ & 0.337 \\
\hline Mean glucose (SD), mg/dL & $138(52)$ & $132(41)$ & $143(58)$ & 0.254 \\
\hline Mean albumin (SD), g/dL & $3.5(0.7)$ & $3.4(0.6)$ & $3.5(0.7)$ & 0.377 \\
\hline Mean $\mathrm{HbA}_{1 \mathrm{c}}$ (SD) & $6.5(1.3)$ & $6.4(1.1)$ & $6.8(1.5)$ & 0.277 \\
\hline Mean WBC (SD), $10^{3} / \mathrm{mm}^{3}$ & $9.1(3.9)$ & $8.8(2.9)$ & $9.3(4.4)$ & 0.488 \\
\hline Median $\mathrm{CHADS}_{2}(\mathrm{IQR})$ & $3(2-4)$ & $3(2-4)$ & $3(2-4)$ & 0.940 \\
\hline Median $\mathrm{CHA}_{2} \mathrm{DS}_{2}$ VASc (IQR) & $4(3-5)$ & $3(3-5)$ & $4(3-5)$ & 0.670 \\
\hline Median NIHSS (IQR) & $5(2-11)$ & $4.5(2-11)$ & $5(2-14)$ & 0.180 \\
\hline Mean serum creatinine (SD), mg/dL & $1.3(0.6)$ & $1.3(0.6)$ & $1.2(0.6)$ & 0.752 \\
\hline \multicolumn{5}{|l|}{ Medication use, $n(\%)$} \\
\hline rt-PA & $4(4)$ & $4(9)$ & 0 & 0.010 \\
\hline Antiplatelet & $43(37)$ & $14(30)$ & $29(41)$ & 0.230 \\
\hline Statin & $65(56)$ & $22(48)$ & $43(61)$ & 0.149 \\
\hline \multicolumn{5}{|l|}{ Final diagnosis } \\
\hline Ischemic stroke, $n(\%)$ & $34(29)$ & $34(74)$ & 0 & - \\
\hline TIA, $n(\%)$ & $12(10)$ & $12(26)$ & 0 & - \\
\hline Hemorrhagic stroke, $n(\%)$ & $24(21)$ & 0 & $24(34)$ & - \\
\hline Mimics, $n(\%)$ & $46(40)$ & 0 & $46(66)$ & - \\
\hline TOAST criteria, $n$ & 34 & 34 & - & - \\
\hline Cardioembolism & 26 & 26 & - & - \\
\hline Lacune & 3 & 3 & - & - \\
\hline Large vessel & 1 & 1 & - & - \\
\hline Undetermined & 4 & 4 & - & - \\
\hline
\end{tabular}

DBP, diastolic blood pressure; INR, international normalized ratio; LDL, low-density lipoprotein; rt-PA, recombinant tissue plasminogen activator; SBP, systolic blood pressure; TIA, transient ischemic attack; WBC, white blood cells. ${ }^{1}$ Arterial indications include atrial fibrillation and antiphospholipid syndrome; venous indications include deep vein thrombosis and pulmonary embolism. 


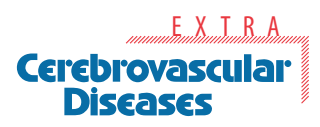

Table 2. Multivariable analysis of likelihood of ischemia

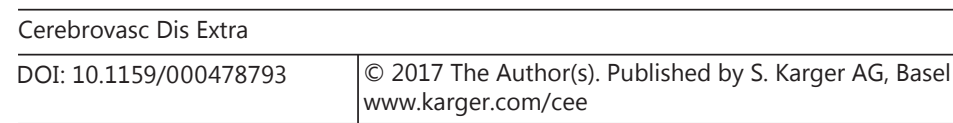

Cao et al.: In Potential Stroke Patients on Warfarin, the International Normalized Ratio Predicts Ischemia

\begin{tabular}{lll}
\hline & $\begin{array}{l}\text { Odds } \\
\text { ratio }\end{array}$ & $\begin{array}{l}95 \% \text { confidence } \\
\text { interval }\end{array}$ \\
\hline $\begin{array}{ll}\text { Entire cohort }(n=116) \\
\text { Age }\end{array}$ & 1.03 & $0.99-1.08$ \\
$\quad$ Race & 1.18 & $0.80-1.73$ \\
Sex & 1.03 & $0.41-2.57$ \\
Initial INR & 0.30 & $0.17-0.55$ \\
$\quad$ Indication for warfarin & 4.11 & $1.33-12.67$ \\
Hemorrhages excluded $(n=92)$ & & \\
$\quad$ Age & 1.04 & $0.99-1.09$ \\
Race & 0.97 & $0.58-1.61$ \\
$\quad$ Sex & 0.84 & $0.29-2.42$ \\
Initial INR & 0.32 & $0.17-0.59$ \\
Indication for warfarin & 4.91 & $1.46-16.57$ \\
\hline
\end{tabular}

INR; international normalized ratio.

divided into two groups: arterial (atrial fibrillation $[n=86]$ and antiphospholipid antibody syndrome $[n=2]$ ) and venous (deep vein thrombosis and pulmonary embolism $[n=28]$ ). Thirty-four patients had ischemic stroke; 12 TIA; 24 hemorrhagic strokes; and 46 stroke mimics. Four patients received thrombolytic therapy (rt-PA) and 1 patient received intraarterial intervention. Common stroke mimics included: seizure $(n=6)$, infection $(n=6)$, dementia/delirium $(n=3)$, hypoglycemia $(n=3)$, recrudescence $(n=4)$, drug toxicity $(n=4)$, systemic impairment $(n=4)$, and functional deficit $(n=2)$.

\section{Initial INR and Ischemic Stroke}

Univariate analysis showed a statistically significant difference in the initial INR for ischemic versus non-ischemic patients (1.7 vs. $2.8, p<0.001$ ). The initial INR values ranged from 1 to 3.4 for ischemic patients versus 1 to 6.4 for those diagnosed with hemorrhagic strokes. Twenty-five percent of hemorrhages were supra-therapeutic and the mean INR was 2.7. Individuals diagnosed with ischemic stroke or TIA were more likely to be on warfarin therapy because of arterial indications, namely atrial fibrillation, compared to non-ischemic patients (87 vs. $67 \%, p=0.024$ ). The cause of stroke was most often cardioembolic (76\%). Aside from a significant difference in platelet number between the ischemic and non-ischemic groups ( 252 vs. $215, p=0.024)$, there were no other differences seen in patient characteristics, including initial National Institute of Health Stroke Scale (NIHSS), CHADS 2 , $\mathrm{CHA}_{2} \mathrm{DS}_{2} \mathrm{VASc}$, and serum creatinine (Table 1). Results were unchanged when patients with hemorrhage $(n=24)$ were removed from analysis.

A multivariable regression was performed with 5 independent variables: age, sex, race, initial INR, and indication for warfarin. Initial INR (OR per 1.0-point increase 0.30 ; $95 \%$ confidence interval [CI] 0.17-0.55) and arterial indication for warfarin (OR 4.11; 95\% CI 1.3312.67) remained statistically significant (Table 2).

Table 3 displays the sensitivity and specificity analysis for INR values. When INR was used to predict ischemia, the area under the curve was high at 0.77 indicating its ability to correctly classify patients. As the INR value increased, the number of patients incorrectly identified as "non-ischemic" significantly decreased (increased specificity). No patient had ischemia with an INR value of 3.6 or greater (specificity 100\%). Predictive values were calculated to determine the likelihood of a patient with a therapeutic INR at $\geq 2$ and $\geq 2.5$ to have a mimic or hemorrhage rather than ischemia. The predictive value for both an INR of $\geq 2$ (sensi- 


\section{Cerebrovascular \\ Diseases}

Table 3. Sensitivity and specificity analysis of INR result

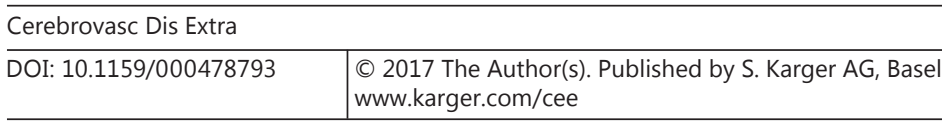

Cao et al.: In Potential Stroke Patients on Warfarin, the International Normalized Ratio Predicts Ischemia

\begin{tabular}{lcc}
\hline INR result & Sensitivity, \% & Specificity, \% \\
\hline$\geq 1$ & 100 & 0 \\
$\geq 1.1$ & 4.35 & 4.35 \\
$\geq 1.2$ & 91.43 & 13.04 \\
$\geq 1.3$ & 85.71 & 34.78 \\
$\geq 1.4$ & 78.57 & 45.65 \\
$\geq 1.5$ & 78.57 & 52.17 \\
$\geq 1.6$ & 75.71 & 58.70 \\
$\geq 1.7$ & 75.71 & 63.04 \\
$\geq 1.8$ & 71.43 & 65.22 \\
$\geq 1.9$ & 68.57 & 67.39 \\
$\geq 2.1$ & 64.29 & 73.91 \\
$\geq 2.2$ & 60.00 & 73.91 \\
$\geq 2.3$ & 51.43 & 78.26 \\
$\geq 2.4$ & 48.57 & 80.43 \\
$\geq 2.5$ & 47.14 & 80.43 \\
$\geq 2.6$ & 42.86 & 86.96 \\
$\geq 2.7$ & 38.57 & 86.96 \\
$\geq 2.8$ & 37.14 & 86.96 \\
$\geq 2.9$ & 32.86 & 86.96 \\
$\geq 3$ & 31.43 & 89.13 \\
$\geq 3.1$ & 30.00 & 97.83 \\
$\geq 3.2$ & 28.57 & 97.83 \\
$\geq 3.3$ & 27.14 & 97.83 \\
$\geq 3.4$ & 24.29 & 97.83 \\
$\geq 3.6$ & 21.43 & 100 \\
$\geq 3.7$ & 20.00 & 100 \\
$\geq 4.3$ & 15.71 & 100 \\
$\geq 4.6$ & 12.86 & 100 \\
$\geq 5.3$ & 11.43 & 100 \\
$\geq 5.4$ & 10.00 & 100 \\
$\geq 5.6$ & 8.57 & 100 \\
$\geq 6.2$ & 5.71 & 100 \\
$\geq 6.4$ & 4.29 & 100 \\
$\geq 9.1$ & 2.86 & 100 \\
$>9.1$ & 0.00 & 100 \\
\hline & &
\end{tabular}

tivity of $69 \%$ and specificity of $67 \%$ ) and an INR of $\geq 2.5$ (sensitivity of $47 \%$ and specificity of $80 \%$ ) was $79 \%$ for a non-ischemic etiology.

\section{Initial INR and Clinical Outcome in Stroke Patients}

The effect of INR result on clinical outcome was examined using discharge mRS. A mRS of $0-2$ was defined as good outcome and a mRS $0-1$ was considered a great outcome. There was a lack of an effect of INR on mRS $<3$ (OR $0.9 ; 95 \%$ CI $0.3-2.5$ ) or mRS $<2$ (OR 4.7; 95\% CI 0.6-35.3) within the ischemic group. In the entire patient population, initial INR also did not affect $\mathrm{mRS}<3$ (OR 0.5 , 95\% CI $0.2-1.1$ ) or $\mathrm{mRS}<2$ (OR 1.1, 95\% CI 0.5-2.5).

\section{Discussion}

Our results indicate that for patients on warfarin presenting with a potential stroke, indication for anticoagulation and INR values are associated with cerebral ischemia. Within our cohort, patients diagnosed with ischemic stroke or TIA were more likely to present with a 
sub-therapeutic INR than those with non-ischemic etiologies in both univariate testing and multivariable regression analyses. Arterial indications for warfarin, mainly atrial fibrillation, further increased the likelihood for ischemic stroke or TIA. This is consistent with prior research. Several studies have shown that a sub-therapeutic INR and atrial fibrillation increase the incidence of ischemic stroke in patients on warfarin with concurrent acute neurologic deficits [11-13]. In addition to showing an association, our data suggests that admission INR can be used to reliably predict the likelihood of ischemia.

The predictive value of INR for ischemic stroke in patients on warfarin who present with acute neurologic symptoms has not been previously well established in literature. The sensitivity and specificity results of this study elucidate the value of INR in this group. When diagnostic imaging does not reveal evidence of hemorrhage or other clear etiology, the INR and indication for anticoagulation may be used in conjunction with pre-test probability (e.g., vascular risk factors) to direct immediate further management. There should be high suspicion for ischemia in individuals with atrial fibrillation who present with a sub-therapeutic INR. Alternatively, the negative predictive value of INR emphasizes the low likelihood of ischemia in patients on warfarin with therapeutic INR values. In our cohort, no ischemia occurred in patients with an INR $\geq 3.6$.

Interestingly, patients who presented with ischemia had a small but statistically significant elevation in platelets compared to those who were not ischemic. Elevated platelets may represent an acute phase reactant $[14,15]$. Advanced age and higher NIHSS scores were not associated with ischemia. This may illustrate that older individuals are at higher risk not only for ischemic stroke but also for other causes of acute neurologic symptoms such as urinary tract infection [16]. While assessing for suspected stroke, physicians' attention should also be given to identifying any clinical or laboratory signs of stroke mimics [15].

We also evaluated the association between INR and patient outcome in our stroke patients using mRS at discharge. We hypothesized that patients with a therapeutic INR would have better outcomes than those with a non-therapeutic INR because emboli may break into smaller pieces, leading to smaller infarcts and less severe strokes in those on anticoagulation. Wakita et al. [17] showed that individuals who are anticoagulated do tend to have smaller infarcts. We found no such association despite that, as expected, the majority of our population had cardioembolic strokes due to atrial fibrillation [18]. There are several possible explanations including our relatively small sample and the numerous medical comorbidities of our population (Table 1) that may have negatively impacted the outcome. It is also possible that a larger difference in mRS would have been observed 90 days after the stroke, allowing time for divergence of the recovery curves for varying stroke severity. Many previous trials found that therapeutic INR was associated with more favorable stroke outcomes in patients with cardioembolic stroke types [19,20], though some failed to detect such a correlation [21]. A larger prospective trial including infarct size and longer-term follow-up is needed.

Our study is not without limitations. The patient population was from a single stroke center - with a relatively small sample size and the majority of patients above the age of 75 years, white, and at high risk for ischemic stroke give their numerous vascular risk factors. The relatively small sample size may have limited our ability to find statistically significant results, and our sensitivities and specificities were based on the prevalence of stroke within our population and therefore our results may not be generalizable to all other populations. In addition, given the retrospective nature, we relied on physician notes and test results within the electronic medical record to determine final diagnosis. Fortunately, the underlying etiology was clear in the majority of cases and any questions were reviewed by a stroke neurologist to ensure that the cases most likely represented mimics rather than ischemic strokes. Finally, the INR can be influenced by numerous factors (foods, medications) and the value on presentation represents only a single data point rather than coagulability over time. 
A patient may have been sub-therapeutic for several days at home but present with a single therapeutic INR. In spite of this, we feel that our data support that admission INR is a fairly good indicator of likelihood of ischemia and may have important clinical applications.

\section{Conclusions}

This study illustrates the usefulness of both INR values and the indication for anticoagulation, specifically atrial fibrillation, in determining the likelihood of ischemic stroke in patients on anticoagulation presenting to the Emergency Department with an acute onset of neurologic symptoms. A sub-therapeutic INR in a patient with atrial fibrillation should significantly increase the suspicion for ischemia; while a supra-therapeutic INR, or lack of atrial fibrillation, is more reassuring that symptoms are more likely due to a mimic. While it is not our intention for a laboratory test to replace physician acumen, it may help to more accurately risk stratify patients and determine the need and urgency of further neurologic imaging and workup.

\section{Disclosure Statement}

The authors declare no conflicts of interest.

\section{References}

1 Mozaffarian D, Benjamin EJ, Go AS, Arnett DK, Blaha MJ, Cushman M, et al; American Heart Association Statistics Committee and Stroke Statistics Subcommittee: Heart disease and stroke statistics - 2015 update: a report from the American Heart Association. Circulation 2015;131:e29-e322.

-2 Lees KR, Bluhmki E, von Kummer R, Brott TG, Toni D, Grotta JC, Albers GW, Kaste M, Marler JR, Hamilton SA, Tilley BC, Davis SM, Donnan GA, Hacke W; ECASS, ATLANTIS, NINDS and EPITHET rt-PA Study Group, Allen K, Mau J, Meier D, del Zoppo G, De Silva DA, Butcher KS, Parsons MW, Barber PA, Levi C, Bladin C, Byrnes G: Time to treatment with intravenous alteplase and outcome in stroke: an updated pooled analysis of ECASS, ATLANTIS, NINDS, and EPITHET trials. Lancet 2010;375:1695-1703.

-3 The National Institute of Neurological Disorders and Stroke rt-PA Stroke Study Group: Tissue plasminogen activator for acute ischemic stroke. N Engl J Med 1995;333:1581-1587.

-4 Hacke W, Kaste M, Bluhmki E, Brozman M, Dávalos A, Guidetti D, Larrue V, Lees KR, Medeghri Z, Machnig T, Schneider D, von Kummer R, Wahlgren N, Toni D: Thrombolysis with alteplase 3-4.5 h after acute ischemic stroke. N Engl J Med 2008;359:1317-1329.

5 Jauch EC, Saver JL, Adams HP Jr, Bruno A, Connors JJ, Demaerschalk BM, et al; American Heart Association Stroke Council, Council on Cardiovascular Nursing, Council on Peripheral Vascular Disease, Council on Clinical Cardiology: Guidelines for the early management of patients with acute ischemic stroke: A guideline for healthcare professionals from the American Heart Association/American Stroke Association. Stroke 2013; 44:870-947.

6 Gottesman RF, Alt J, Wityk RJ, Llinas RH: Predicting abnormal coagulation in ischemic stroke: reducing delay in rt-PA use. Neurology 2006;67:1665-1667.

7 Holbrook A, Schulman S, Witt DM, Vandvik PO, Fish J, Kovacs MJ, Svensson PJ, Veenstra DL, Crowther M, Guyatt GH: Evidence-based management of anticoagulant therapy: antithrombotic therapy and prevention of thrombosis, 9th ed: American College of Chest Physicians Evidence-Based Clinical Practice Guidelines. Chest 2012; 141:e152S-e184S.

-8 Baker WL, Cios DA, Sander SD, Coleman CI: Meta-analysis to assess the quality of warfarin control in atrial fibrillation patients in the United States. J Manag Care Pharm 2009;15:244-252.

9 Schein JR, White CM, Nelson WW, Kluger J, Mearns ES, Coleman CI: Vitamin K antagonist use: evidence of the difficulty of achieving and maintaining target INR range and subsequent consequences. Thromb J 2016;14:14

10 Reynolds MW, Fahrbach K, Hauch O, Wygant G, Estok R, Cella C, Nalysnyk L: Warfarin anticoagulation and outcomes in patients with atrial fibrillation: a systematic review and metaanalysis. Chest 2004;126:19381945.

11 Wolf PA, Abbott RD, Kannel WB: Atrial fibrillation as an independent risk factor for stroke: the Framingham Study. Stroke 1991;22:983-988. 
12 Longstreth WT Jr, Bernick C, Fitzpatrick A, Cushman M, Knepper L, Lima J, Furberg CD: Frequency and predictors of stroke death in 5,888 participants in the Cardiovascular Health Study. Neurology 2001;56:368-375.

$>13$ Hylek EM, Go AS, Chang Y, Jensvold NG, Henault LE, Selby JV, Singer DE: Effect of intensity of oral anticoagulation on stroke severity and mortality in atrial fibrillation. N Engl J Med 2003;349;1019-1026.

-14 Jaremo P, Erksson M, Lindahl TL, Milovanovic M: Platelets and acute cerebral infarction. Platelets 2013; 24:407-411.

15 Fateh-Moghadam S, Htun P, Tomandl B, Sander D, Stellos K, Geisler T, et al: Hyperresponsiveness of platelets in ischemic stroke. Thromb Haemost 2007;97:974-978.

-16 Kose A, Inal T, Armagan E, Kiyak R, Demir AB: Conditions that mimic stroke in elderly patients admitted to the emergency department. J Stroke Cerebrovasc Dis 2013;22:e522-e527.

17 Wakita M, Yasaka M, Minematsu K, Yamaguchi T: Effects of anticoagulation on infarct size and clinical outcome in acute cardioembolic stroke. Angiology 2002;53:551-556.

18 Adams HP Jr, Bendixen BH, Kappelle LJ, Biller J, Love BB, Gordon DL, Marsh EE 3rd: Classification of subtype of acute ischemic stroke. Definitions for use in a multicenter clinical trial. TOAST. Trial of Org 10172 in Acute Stroke Treatment. Stroke 1993;24:35-41.

19 Nakamura A, Ago T, Kamouchi M, Hata J, Matsuo R, Kuroda J, Kuwashiro T, Sugimori H, Kitazono T; Fukuoka Stroke Registry Investigators: Intensity of anticoagulation and clinical outcomes in acute cardioembolic stroke: the Fukuoka Stroke Registry. Stroke 2013;44:3239-3242.

-20 Indredavik B, Rohweder G, Lydersen S: Frequency and effect of optimal anticoagulation before onset of ischaemic stroke in patients with known atrial fibrillation. J Intern Med 2005;258:133-144.

21 Paciaroni M, Agnelli G, Caso V, Venti M, Alberti A, Milia P, Silvestrelli G, Biagini S: Prior use of antithrombotic agents and neurological functional outcome at discharge in patients with ischemic stroke. J Thromb Haemost 2006;4:1957-1961. 